\title{
Review
}

\section{MANAGEMENT OF HEALTH RISK RELATED TO USE OF ENGINEERED NANOMATERIALS. AN ANALOGY WITH BIOSAFETY}

\author{
Dimiter Prodanov \\ Environment, Health and Safety, Imec, Belgium
}

\begin{abstract}
Safety of nanomaterials is a new scientific field, which draws increasing attention in literature. Among the challenges the field is facing are the insufficient amount and quality of nanotoxicological data and the ambiguity in the metrics describing the exposure. This results in substantial difficulties in the actual quantification of risk in terms of dose-response relationships and exposure limits, which is a cornerstone of chemical risk assessment. While there is no golden standard for risk assessment and management several pragmatic systems have come into being. All of these employ some form of categorization and grouping of materials into hazard groups. The present review aims to draw analogies between the nascent field of nanosafety and the well established field of biosafety, where the risk is also difficult to quantify. Biomed Rev 2017; 28:100-104.
\end{abstract}

\section{NANOMATERIALS}

The nanomaterials are broadly defined as those materials that have a certain percentage of particles at the nanoscale between 1 and $100 \mathrm{~nm}$ (see below). Particles of this size can get deposited in the lungs, pass easily through alveoli or even disperse in the body. While the size cut-off is somehow arbitrary it nevertheless conveys the important fact that properties of the features at nanoscale (i.e. nanoforms) can substantially differ from the properties of "macro" materials in bulk. Such desirable characteristics can include increased strength of the material, its chemical reactivity or altered electrical properties. These features offer possibilities for new applications in a broad range of sectors; for example in medicine (e.g. detection of genetic sequences using DNAtagged gold nanoparticles); environment (e.g. waste-water treatment with carbon nanotube filters); or energy production (e.g. solar cells using silicon nanocrystals). At the same time, the use of manufactured nanomaterials in a number of commercial applications raises questions regarding potential unintended risks for the workers, consumers and the environment.
Top-down and bottom-up approaches to nanotechnology Nano-enabled technologies have been with mankind for centuries, if not millennia. What, however, makes modern nanotechnology unique is the aspect of deliberate exploitation of the nanoscale. Nanotechnology uses intentionally desirable properties of materials at the nanoscale to deliver economically beneficial effects. From technological perspective there are two complementary approaches: top-down and bottom-up processing. While the bottom-up approach can be broadly identified as particle synthesis, or self-assembly; top-down approach can be thought of growth or deposition on surfaces. An example of the latter is micro or nano-electronics industry. Nanomaterial manufacturers typically employ bottom-up approaches, while end-user industries incorporate engineered nanomaterials on macroscopic objects, e.g. products like computer chips, tires, sportswear. From industrial end-user perspective the innovation cycle leads to new materials and nanoforms being introduced in manufacturing. Typically, the product development runs ahead of establishing metrics for the occupational and environmental hazards of these materials. Examples include nanoelctronics, automotive, cosmetics and painting industries.

Received 24 November 2017, revised 6 December 2017, accepted 7 December 2017.

Correspondence and reprint request to Dimiter Prodanov, Kapeldreef 75, 3001, Leuven, Belgium. Tel: +32 162818 40;

E-mail: dimiter.prodanov@imec.be; dimiterpp@gmail.com 


\section{INTERNATIONAL EFFORTS AND EU INITIATIVES IN NANOSAFETY RESEARCH}

Traditional chemical risk assessment tools are based on a quantitative dose-response relationship, which is augmented by setting a threshold defining the noeffect levels, or occupational exposure limit (OEL). This type of risk assessment is applicable in cases when sufficient toxicological and occupational exposure data are available. Understanding properties of engineered nano-materials and how they affect living systems, such as the human body, draws substantial attention in recent scientific literature (Fig. 1). While the predominant number of studies are focused on the materials, relatively smaller amounts discuss more comprehensive safety questions.

It becomes increasingly clear that harmful properties of nanoforms sometimes do not correlate with the toxicological profile of the bulk materials. There are several reasons for that, notably the change of scale leads to a relative increase of the fraction of particles which are on the surface of the nanoobject. The curvature and charge density also increase with the decrease of size. One of the challenges in nano-toxicology is therefore, the identification of the best metric for toxicological assessment (11). While traditional metrics are based on mass concentration, the majority of authors at present favor particle number based metrics.

A related challenge is how a nanomaterial can be defined as there are several different definitions internationally. According to the current recommendation by the European Commission a nanomaterial means:

A natural, incidental or manufactured material containing particles, in an unbound state or as an aggregate or as an agglomerate and where, for $50 \%$ or more of the particles in the number size distribution, one or more external dimensions is in the size range $1 \mathrm{~nm}-100 \mathrm{~nm}$.

In specific cases and where warranted by concerns for the environment, health, safety or competitiveness the number size distribution threshold of $50 \%$ may be replaced by a threshold between 1 and $50 \%$. By derogation from the above, fullerenes, graphene flakes and single wall carbon nanotubes with one or more external dimensions below $1 \mathrm{~nm}$ should be considered as nanomaterials.

On the other hand, ISO defines a nano-object simply as "material with one, two or three external dimensions in the nanoscale", that is between 1 and $100 \mathrm{~nm}$. To address so-outlined scienfic and regulatory challenges international bodies, such as the European Union and the Organisation for Economic Co-operation and Development (OECD), have orchestrated substantial efforts. For example, the EU NanoSafety Cluster (www.nanosafetycluster.eu) is a network of projects in the funding programs FP6 (2002-2006), FP7 (2007-2013), and H2020 (2014-2020) framework programmes, intended at harmonizing the European Commission-funded in nanosafety research. Over that time, more than 50 projects have been associated with the NanoSafety Cluster.

Challenges in the risk assessment approach can be traced to the assumption that the hazard and the risk can be quantified. From biosafety practice, we know that this may not (ever) be the case. A holistic view of the nano-hazard and the related process risk is crucial for successful integration of nanosafety aspects into the overall risk management methodology. Material properties, health effects, potential of release and occupational exposure are principal aspects for success- 


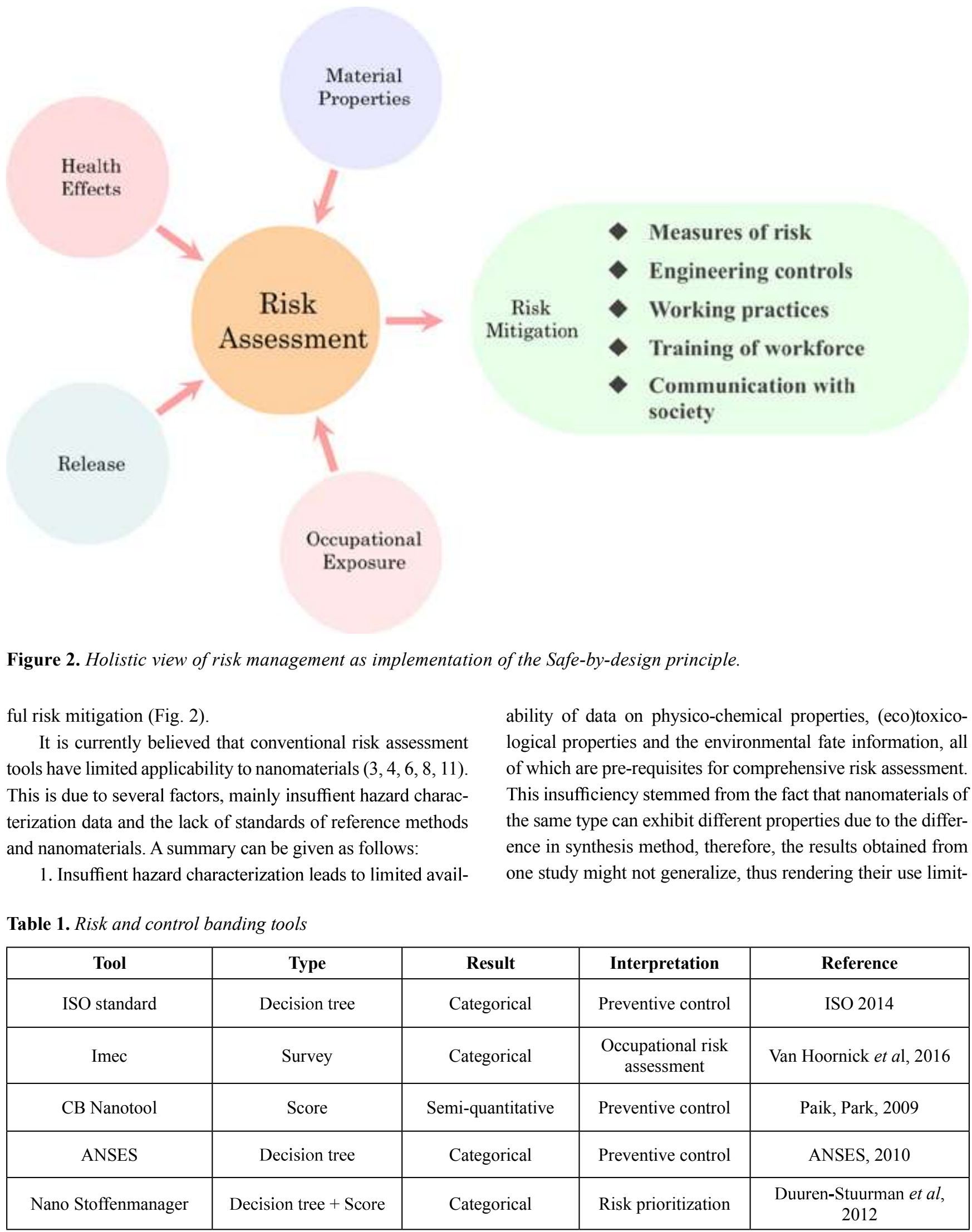


Table 2. Hazard grouping according to ISO/TS 12901

\begin{tabular}{|c|c|c|}
\hline ISO Hazard class & Definition & Examples \\
\hline Category A & No significant risk to health & Non-toxic soluble nanoparticles \\
\hline Category B & Slight Hazard - Slightly toxic & - \\
\hline Category C & Moderate Hazard & - \\
\hline Category D & Serious hazard & $\begin{array}{c}\text { Carciongenic insoluble nanoparticles, } \\
\text { insoluble fibers, such as carbon nanotubes }\end{array}$ \\
\hline Category E & Severe hazard & \\
\hline
\end{tabular}

ted $(3,4,6)$.

2. Poorly designed experimental protocols can lead to false positive or negative toxicological results because nanoparticles have been shown to directly interfere with the biological/toxicological assays used. Recent publications have demanded for a uniform standard operating procedures so that results obtained from different studies can be comparable and that a more concrete conclusion can be drawn $(6,8)$.

3. Additionally, due to the lack of data, occupational exposure limits (OELs) for most types of nanoparticles are unknown (NIOSH 2009; 11). The OELs for bulk parent materials can significantly differ from their nano counterparts due to the unique physico-chemical properties exhibted in the nano form and therefore, the two OELs are not interchangeable.

4. The best metric (by mass, concentration or number) for nanoparticles exposure characterization is still debatable. While there is no golden standard for risk assessment and management several pragmatic systems have come into being. So identified gaps in understanding promote the view that the most promising approaches for management of nano-related risk are the risk and control- banding tools (Table 1), which are akin to the biohazard grouping. Efforts in the direction of grouping of nanomaterials and read-across missing properties of nanoforms are encouraged by the stakeholders, such as the nanotechnology industry, national regulators and international bodies, like OECD. There is a recent in-

Table 3. Biohazard classification according to EU Directive 2000/54 and WHO. Examples are given according to the Belgian state regulations. AAV - Adeno-associated virus, HBV-Hepatitis B Virus, HCV-Hepatitis C Virus, HIV-Human Immunodeficiency Virus

\begin{tabular}{|c|l|c|}
\hline Biohazard class & \multicolumn{1}{|c|}{ Definition } & Examples \\
\hline Group 1 & Biological agent that is unlikely to cause human disease. & E. coli K 12 strain, AAV \\
\hline Group 2 & $\begin{array}{l}\text { Biological agent that can cause human disease and } \\
\text { might be a hazard to workers; it is unlikely to spread to } \\
\text { the community; there is usually effective prophylaxis or } \\
\text { treatment available. }\end{array}$ & $\begin{array}{c}\text { Most strains of Influenza } \\
\text { A virus }\end{array}$ \\
\hline Group 3 & $\begin{array}{l}\text { Biological agent that can cause severe human disease } \\
\text { and present a serious hazard to workers; it may } \\
\text { present a risk of spreading to the community, b u t } \\
\text { there is usually effective prophylaxis or treatment } \\
\text { available. }\end{array}$ & HBV, HCV, HIV \\
\hline Group 4 & $\begin{array}{l}\text { Biological agent that causes severe human disease and } \\
\text { risk of spreading to the community; present a high } \\
\text { no effective prophylaxis or treatment available. }\end{array}$ & Ebola virus, \\
\hline
\end{tabular}


ternational standard dealing with nanosafety in occupational settings: ISO Technical Standard ISO/TS 12901-2:2014 "Nanotechnologies - Occupational risk management applied to engineered nanomaterials". The standard is focused on intentionally produced nano-objects, such as nanoparticles, nanopowders, nanotubes, nanowires, as well as of aggregates and agglomerates and is focused on the management of the inhalation hazard. The standard defines 5 nanohazard groups which combine with four exposure bands (Table 2). This is constrained by 5 control bands, which identify measures to be implemented in order to reduce the risk for the personnel.

\section{WHAT CAN WE LEARN FROM BIOSAFETY?}

In biosafety practice, there are 4 biohazard groups and 4 major biocontainment levels (Table 2). Biosafety levels are based on a composition of facility design features, containment infrastructure, specialized equipment, work practices and operational procedures required for working with agents from the four risk groups (13). The emphasis is on the classification of biological agents based on the similarity of their action on a healthy human being. That is the risk is measured in a relative way! This pragmatic classification provides the backbone for management of biorisk in laboratory setting for more than 20 years.

The current expert opinion identifies grouping criteria as priority in nanosafety research (11). In contrast to biosafety practice, in nanotechnology, each step of a process where nanomaterials are used or produced is assigned a particular risk level, which in turn determines the appropriate risk control measures. In my view, there are a number of synergies between both fields that can be exploited for cross-fertilization of the efforts. This is an open field of research, which may lead us out of the maze of conflicting nanotoxicology reports.

\section{CONFLICT OF INTEREST STATEMENT}

The author certifies that he has no any financial interest in the subject matter discussed in the present review.

\section{ACKNOWLEDGEMENTS}

The work is supported by the NanoStreeM project, funded under H2020 grant agreement 688194 of the European Commission.

\section{REFERENCES}

1. ANSES. Development of a Specific Control Banding Tool for Nanomaterials. 2010.
2. ANSES. Assessment of the risks associated with nanomaterials: Issues and update of current knowledge. 2014. https:/www.anses.fr/en/content/assessment-risks-associated-nanomaterials.

3. Brouwer D, van Duuren-Stuurman B, Berges $M$, Jankowska E, Bard D, Mark D. From workplace air measurement results toward estimates of exposure? Development of a strategy to assess exposure to manufactured nano-objects, J Nanopart Res 2009; 11, 1867. DOI: $10.1007 / \mathrm{s} 11051-009-9772-1$.

4. Brouwer D. Control banding approaches for nanomaterials, Ann Occup Hyg 2012; DOI: 10.1093/annhyg/ mes039.

5. Corlan D. Medline trend: automated yearly statistics of PubMed results for any query. 2004. Web resource at URL:http://dan.corlan.net/medline-trend.html.

6. Dekkers S, Oomen AG, Bleeker EA, Vandebriel RJ, Micheletti C, Cabellos J, et al. Towards a nanospecific approach for risk assessment. Regul Toxicol Pharmacol 2016; 80: 46-59. DOI: 10.1016/j.yrtph.2016.05.037.

7. Duuren-Stuurman BV, Vink SR, Verbist KJM, Brouwer HGAHDH, Kroese DED, Niftrik MFJV, et al. Stoffenmanager Nano Version 1.0: A Web-Based Tool for Risk Prioritization of Airborne Manufactured Nano Objects. Ann Occup Hyg 2012; DOI: 10.1093/annhyg/ mer113.

8. Hristozov D, Gottardo S, Semenzin E, Oomen A, Bos P, Peijnenburg W, et al. Frameworks and tools for risk assessment of manufactured nanomaterials, Envir Int 2016; 95: 36-53. DOI: 10.1016/j.envint.2016.07.016.

9. NIOSH. Approaches to Safe Nanotechnology. Managing the Health and Safety Concerns Associated with Engineered Nanomaterials, DHHS (NIOSH) Publication No. 2009-125, 2009.

10. Paik U, Park J-G. Nanoparticle engineering for chemical-mechanical planarization: Fabrication of next-generation nanodevices. CRC Press 2009.

11. Savloaainen $\mathrm{K}$, et al. Towards safe and sustainable nanomaterials and nanotechnology innovations, Finnish Inst Occup Health (Helsinki) 2013. DOI: 10.13140/2.1.3084.8969.

12. Van Hoornick N, Prodanov D, Pardon A. Banding approach for engineered nanomaterial risk assessment and control. J Phys: Conference Series 2017; 838: 012017. DOI: 10.1088/1742-6596/838/1/012017.

13. Laboratory Biosafety Manual, WHO 2004. 